\title{
Design of a Wideband Band-Pass Filter Using Semi-lumped and Semi-distributed Technology
}

\author{
Ying $\mathrm{Liu}^{1}$, Jiayu Xie ${ }^{1}$, Junling Huang ${ }^{1}$ \\ ${ }^{1}$ Aviation Engineering Institution, Civil Aviation Flight University of China Guanghan, Sichuan, \\ 618307, China
}

Keywords: UHF. wideband. Miniaturize. Bandpass filter

\begin{abstract}
In order to design a miniaturized wideband broadband band-pass filter in UHF band, this paper develops a method by cascading a lumped parameter highpass filter with another distribution parameter lowpass filter. Detailed design and synthesis procedures are provided; moreover, agreement between measured and theoretically predicted results demonstrates feasibility of the proposed filter. Due to its simple structure, the UHF wideband band pass filter newly introduced in this paper is suitable for implementation on printed circuit boards. After processed, the size is smaller than $32 \times 15 \mathrm{~mm} 2$.
\end{abstract}

\section{Introduction}

In recent years, there are increasing needs for broader bandwidth in UHF wireless communication systems; moreover, much research has focused on wideband filters design. The bandpass filter, providing a low insertion loss in the band and attenuation out of the band, plays a very important role in the RF system. Just because the UHF band belongs to low microwave band, correspondingly, the size requirement of the filter will be as small as possible. Various methods to make wideband filters are reviewed as following.

There proposed two cascading low-pass filters with high-impedance line $e^{[1]}$. This class has a disadvantage. When the cutoff frequency of a lowpass filter is a farther from the previous one, rectangular factor improvement is not obvious. Conversely, it is difficult to completely suppress spurious frequency. The lowpass filter with issuing lines and stepped-impedance resonators, without parallel-coupled, can improve the rectangular factor, and broaden the width of barrier ${ }^{[2]}$. Nevertheless, circuit size are not satisfying because it has a larger horizontal geometry size. Proposed wideband filter cannot be practically implemented in a wideband system.

Considering the advantages and disadvantages of these two filter units, many issuing lines and stepped impedance resonators (SIR) will be led into the parallel-coupled resonators in this article. This structure can realize function of the lowpass filter. Highpass filter uses lumped parameter distribution components.

Due to the trend toward broader bandwidth and better selectivity, filters with a wide stopband in a linear wideband system are promising. In order to achieve this goal, this paper adopts lumped parameter highpass filter with another distribution parameter lowpass filter to achieve the bandpass filter. Moreover, with properly selected transmission-line impedance, highpass filter can be easily achieved. In particular, this type filter can also minimize the size of the bandpass filter.

Following are detailed procedures for filter development. Sections II and III present design procedures for the broadband lowpass filter and the broadband highpass filter, respectively. Consequently, Section IV introduces the integration of the newly developed wideband bandpass filter. Finally, this paper is concluded in Section V.

\section{Design of broadband lowpass filter}

In this paper, the design of low-pass filter is interpreted as the multi parallel-coupled resonator loading multi-order elliptic function low-pass filter structure. It uses the parallel-coupled resonator to suppress spurious frequency, which produces stopband transmission zeros configuration. Set up the 
structure of each unit to make its transmission zero close to each other. With the order of elliptic function filter increasing, the stopband rejection degree and the rectangular factor will be always significantly improved. This type has more advantages on the improvement of the rectangular factor.

In order to reflect the advantages of parallel-coupled SIR in hairpin lowpass filter, there is two filers' frequency response compared. They are shown in Figure 1. Fig 1(a) is not loading hairpin parallel coupled line low-pass filter, fig 1(b) is equivalent LC model, fig 1(c) is loading the hairpin parallel coupled line low-pass filter, fig 1(d) is equivalent LC model, and both of frequency responses can be seen in fig 1(e).

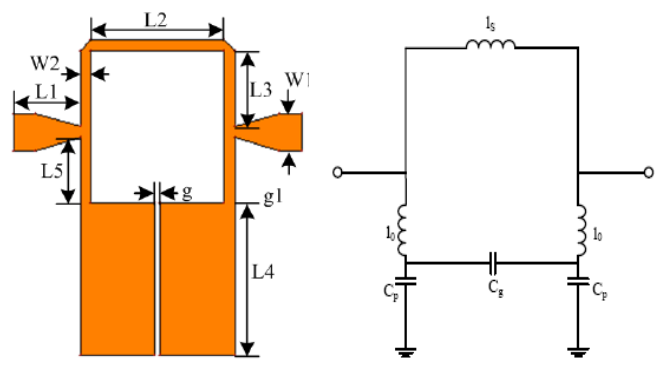

(a)

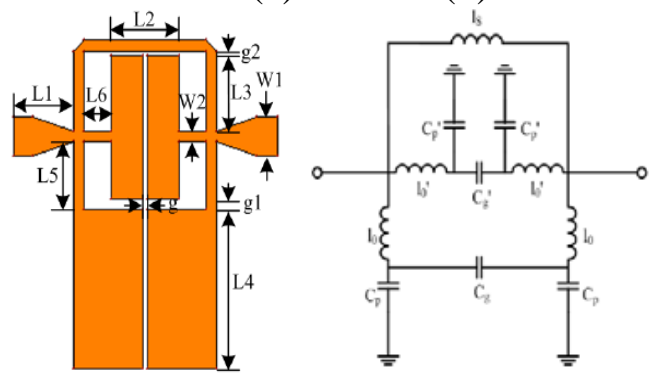

(c)

(d)

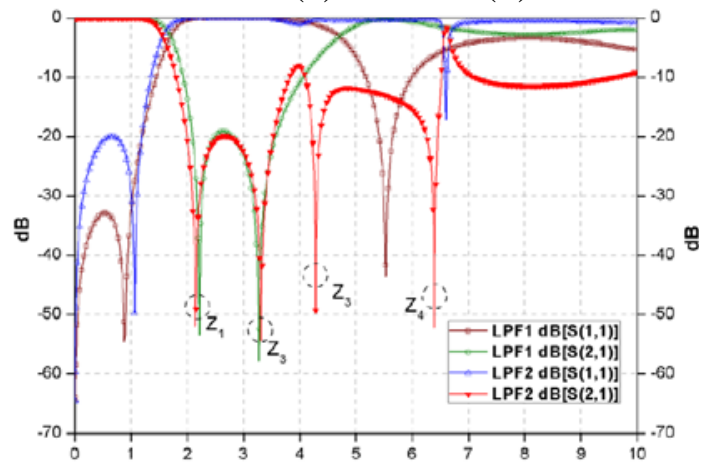

(e)

Fig. 1 Two lowpass filters

Fig.e shows that LPF1 doesn't load parallel coupled lowpass filter, and LPF2 loads parallel coupled lowpass filter. It can be found that: when the filter loads parallel-coupled SIR resonator, there are two new transmission zeros Z3 and Z4. They are far from two transmission zeros Z1 and Z2 in the original structure. Appropriately adjust positions of transmission zero Z3 and Z4, and it can offset the impact of spurious frequency transmission pole to stopband, and also can increase the resistance width.

From the analysis in INTRODUCE, this paper adopts a parallel-coupled SIR resonator loading multi-order hairpin line step-impedance lowpass filter structure. At the same time, in order to make the first stopband transmission zero move towards low-end frequency, it adopts interdigital coupled method to increase coupling capacitance value, moreover, it can further improve the rectangular factor, which doesn't affect the passband characteristics. The elementary design of the lowpass filter is shown in Figure 2. 


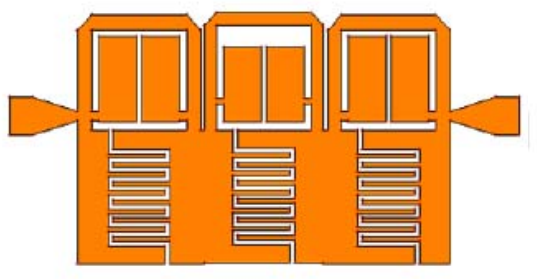

(a) circuit structure

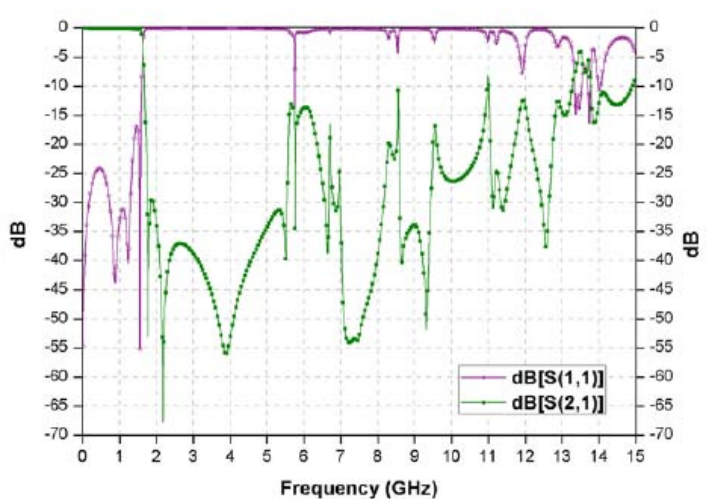

(b) the frequency response curve

Fig. 2 The first lowpass filter

It can be found that the reflection coefficient in the passband is a little high, and when the stopband frequency is more than $5.5 \mathrm{GHz}$, the stopband attenuation is not good enough. Therefore, according to the law of the zero adjustment, it should be optimized in the simulation software. Finally, parallel coupled SIR loading multi-order elliptic function lowpass filter is shown in Figure 3.

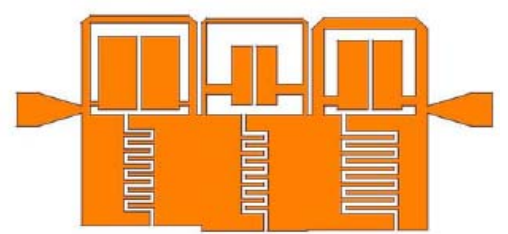

(a) circuit structure

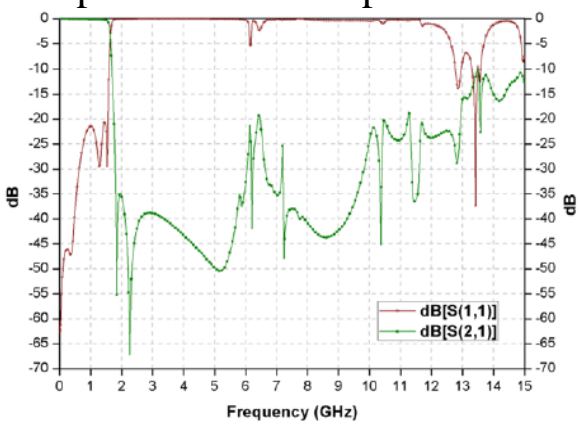

(b) the frequency response curve

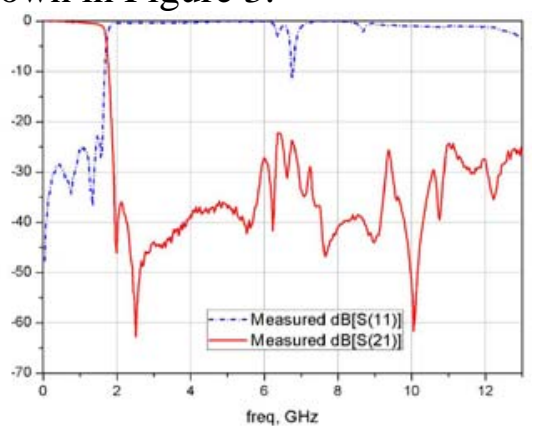

(c) the measured curve

Fig. 3 The final lowpass filter

Then, it is machine processed, and tuned. So the measured frequency response curves are gotten. and shown in Fig 3(c). The reflection coefficient and the stopband rejection have been improved.

In this section, it proposes a parallel-coupled SIR loading hairpin multi-order elliptic function low-pass filter structure. This type has wider stopband, a better rectangular factor, and smaller size.

\section{Design of broadband highpass filter}

In order to avoid design difficulty of miniaturization filter in low microwave band, this paper uses lumped elements to design the high-pass filter. This paper uses Design Guide in the ADS simulation software to complete the required high-pass filter design, as shown in Figure 4. Taking the impact of parasitic parameters of the high frequency components into account, the designed highpass filter has much attention, just like: firstly choose less inductor topological structure, try to use the capacitance to absorb the parasitic capacitance, and choose smaller discrete values in the circuit. 


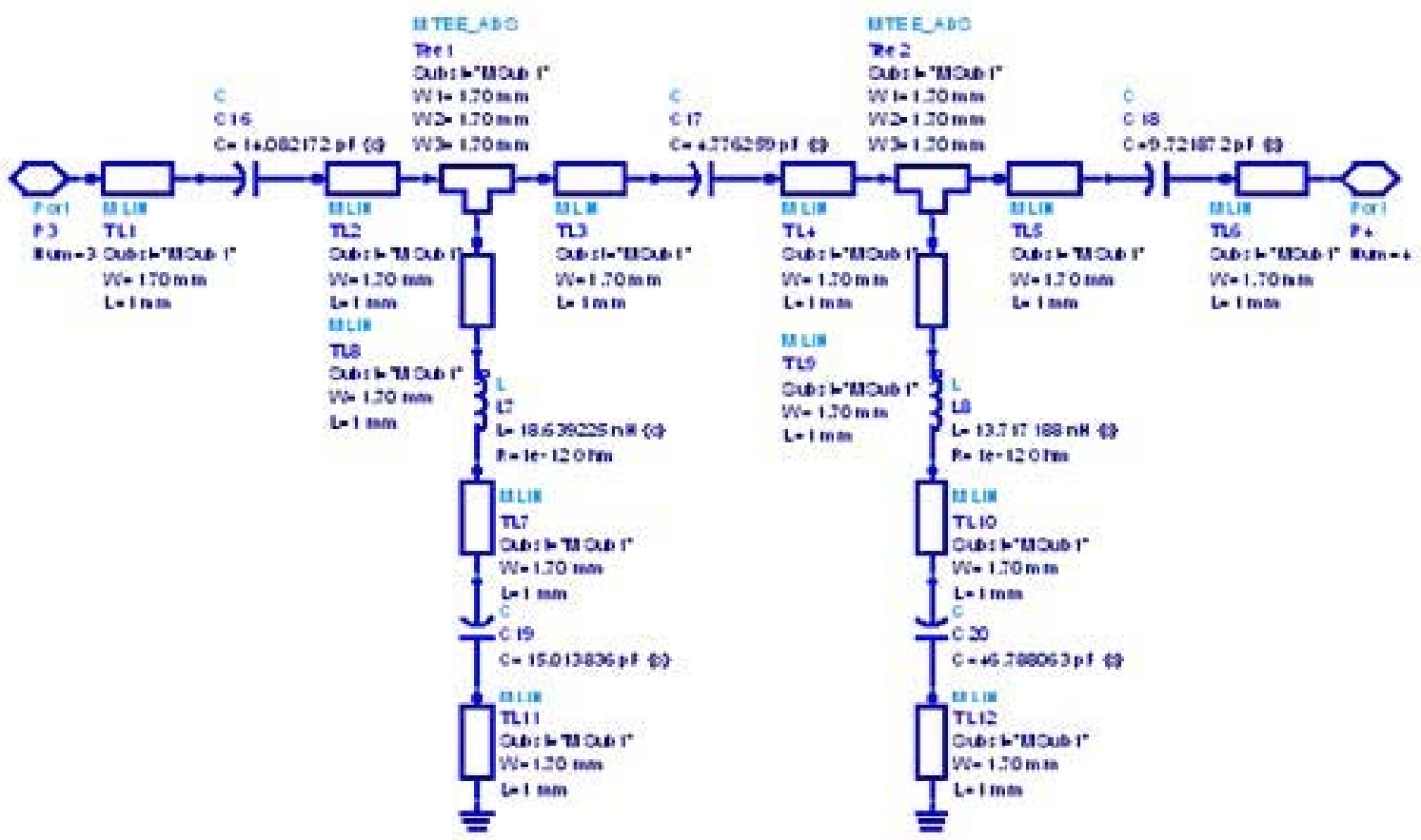

Fig. 4 The schematic diagram of the highpass filter

Usually, the index requirement is set more highly than the actual need for performance, and then optimized in the range of discrete values, so the highpass filter is gotten. However, it should be not machine processed with an actual object. Just because it is as a part circuit of the bandpass filter. After connecting highpass filter and lowpass filter, it should be optimized to ensure that the bandpass filter has good performance whether in band or out of the band. Here, this paper designs the connection pads, optimize the ideal component values. And last, the highpass filter will be as a whole to design the bandpass filter. So here, the bandpass filter will be directly on design.

\section{Design of broadband bandpass filter}

Based on the parallel-coupled SIR loading multi-order elliptic function lowpass filter and ideal circuit topology of highpass filter, this paper designs broadband bandpass filter which has good performance. First of all, measures S-parameters of the parallel coupled SIR loading the multi-order elliptic function lowpass filter, and generate an S2P file. Then, in the ADS simulation design software, the highpass filter adds the S2P file, and then optimize to get the UHF band broadband bandpass filter. Last, machine processed, and tuned. The actual object size is $32 * 15 \mathrm{~mm}^{2}$.The CAD layout and measured parameters are shown in figure 5.

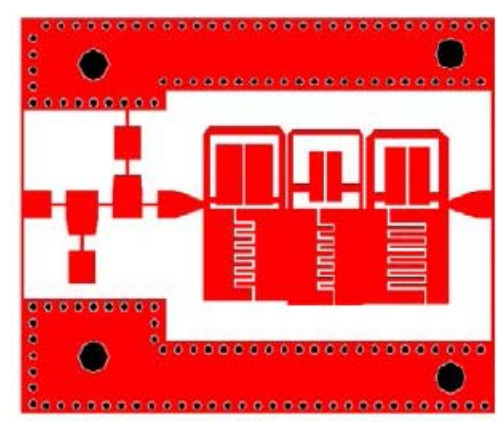

(a) CAD layout

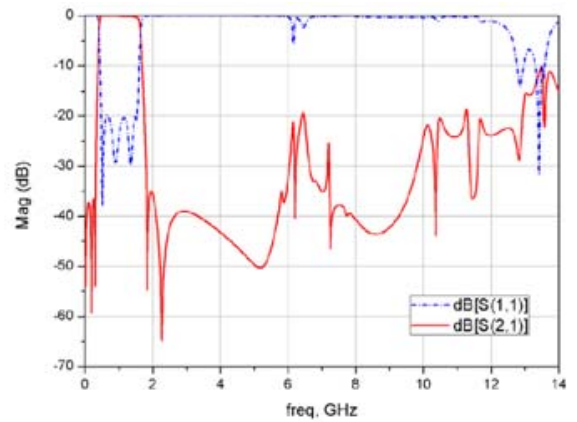

(b) the theoretical results

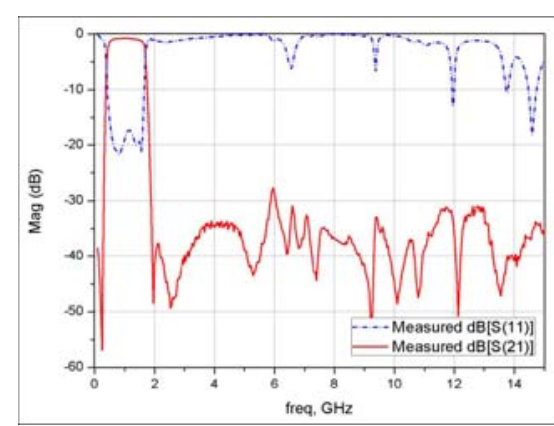

(c) the measured results

Fig. 5 The bandpass filter

Fig 5(b) indicates that: in the band range, insertion loss is less than $1.2 \mathrm{~dB}$, and the reflection coefficient is also less than $-16 \mathrm{~dB}$. At the same time, the band rejection is greater improvement out of the band. And it has a good wide stopband characteristic. Compared to the design of low-pass filter unit, band pass filter of wide stopband characteristics is much better, mainly because lumped 
elements are introduced. It can change the resonant response of the overall structure, and weaken the impact of the spurious frequency, which broadens the width resistance of the band-pass filter.

\section{Conclusion}

A UHF broadband bandpass filter is developed in this paper by cascading a highpass filter and lowpass filter. By properly design, the highpass filter and lowpass filter are gotten. Moreover, agreement between theoretical results and measured results validates the proposed structure. Due to its simple structure and attractive performance, the newly proposed filter is appropriate for implementation in the UHF wideband systems.

\section{Reference}

[1] Sha Luo, Lei Zhu,Sheng Sun: Stopband-Expanded Low-Pass Filters Using Microstrip Coupled-Line Hairpin Units. IEEE Microwave and Wireless complements letters, August 2008,Vol.18,NO.8:506 508

[2] Lung-Hwa Hsieh, Kai Chang: Compact Elliptic-Function Low-pass Filter Using Microstrip Stepped-Impedance Hairpin Resonators. IEEE transactions on microwave theory and techniques, VOL.51,NO.1,January 2003:193 199

[3] M.H. Yang and J.Xu: Design of compact, broad-stopband lowpass filter using modified stepped impedance hairpin resonators, Electronics letters, 2008,44(20)

[4] ULRICH L.Rohde, David P.Newkirk: RF/Microwave Circuit Design for Wireless Applications. New York: John Wiley\&Sons,2000, 565-581 